\title{
Força iônica da solução de equilíbrio na adsorção de arsênio em latossolos brasileiros
}

\author{
Mari Lucia Campos(1), Luiz Roberto Guimarães Guilherme ${ }^{(2)}$, Elton Visiolli(2), Alessandra Silveira Antunes ${ }^{(2)}$, \\ Nilton Curi(2), João José Marques ${ }^{(2)}$ e Marx Leandro Naves Silva(2)
}

\begin{abstract}
(1)Universidade do Estado de Santa Catarina, Dep. de Solos, Caixa Postal 281, CEP 88520-000 Lages, SC. E-mail: campos_ml@yahoo.com.br (2)Universidade Federal de Lavras, Dep. de Ciência do Solo, Caixa Postal 37, CEP 37200-000 Lavras, MG. E-mail: guilherm@ufla.br, niltcuri@ufla.br,.jmarques@ufla.br, marx@ufla.br, eltonvisioli@yahoo.com.br, lessi.antunes@bol.com.br
\end{abstract}

\begin{abstract}
Resumo - O objetivo deste trabalho foi avaliar o efeito da força iônica da solução de equilíbrio sobre a adsorção e dessorção de arsênio, em 17 latossolos, de diferentes regiões geográficas brasileiras. A adsorção de As foi avaliada em dois valores de força iônica (15 e $150 \mathrm{mmol} \mathrm{L}^{-1}$ ), duas doses de As (15 e $150 \mu \mathrm{mol} \mathrm{L}^{-1}$ ), relação solo:solução final de 1:100 e pH 5,5. Não houve diferença nas porcentagens de adsorção, entre os valores de força iônica 15 e $150 \mathrm{mmol} \mathrm{L}^{-1}$ (63,51 e 64,46\%). A porcentagem de dessorção na dose $15 \mu \mathrm{mol} \mathrm{L} \mathrm{L}^{-1}$ foi inferior a 15\%, nos dois valores de força iônica, exceto para os solos LVd4 e LAd1, em que a porcentagem de dessorção foi superior a $20 \%$.
\end{abstract}

Termos para indexação: sorção, contaminação química, poluente, elementos-traço.

\section{Ionic strength of the equilibrium solution upon arsenic adsorption in Brazilian Oxisols}

\begin{abstract}
The objective of this work was to evaluate the effect of ionic strength of the equilibrium solution on adsorption and desorption of arsenic, in 17 Oxisols of different Brazilian geographic regions. Arsenic adsorption was evaluated at two values of ionic strength (15 and $\left.150 \mathrm{mmol} \mathrm{L}^{-1}\right)$, two doses of As (15 and $150 \mu \mathrm{mol} \mathrm{L}^{-1}$ ), soil:solution ratio of 1:100 and $\mathrm{pH}$ 5.5. There were no differences in percentages of adsorption between the values of ionic strength 15 and $150 \mathrm{mmol} \mathrm{L}^{-1}$ (63.51 and 64.46\%). Desorption percentage at the dose $15 \mu \mathrm{mol} \mathrm{L}^{-1}$ was smaller than $15 \%$ at the two values of ionic strength, except for LVd4 and LAd1, in which the desorption percentage was higher than $20 \%$.
\end{abstract}

Index terms: sorption, chemical contamination, pollutants, trace elements.

\section{Introdução}

O arsênio foi considerado, pelo sexto ano consecutivo, a substância mais perigosa à saúde humana (United States, 2003). O arsênio é mediana a altamente tóxico para as plantas e altamente tóxico para mamíferos (McBride, 1989). A exposição humana a arsênio pode resultar em desenvolvimento de câncer de pele, de pulmão, de fígado, de bexiga, de rins e de cólon (United States, 2000; Basu et al., 2001). No solo, ele pode ser originário de fontes naturais e de fontes antropogênicas, essa última proveniente de pesticidas, herbicidas, fertilizantes, emitido durante a mineração e fundição do ouro, chumbo, cobre e níquel, produção de ferro e aço, combustão de carvão (Smith et al., 1998; Baird, 2002) e irrigação com água contaminada (Roychowdhury et al., 2002).
No Brasil, a mineração de ouro e ferro tem contribuído para a dispersão de arsênio e sua entrada na cadeia alimentar. Um exemplo disso é sua concentração de $300 \mu \mathrm{g} \mathrm{L}^{-1}$ em águas superficiais o teor máximo, indicado pela portaria 1496, da Funasa, em 2000, é de $10 \mu \mathrm{g} \mathrm{L}^{-1}-$, de 20 a $4.000 \mathrm{mg} \mathrm{kg}^{-1}$ em sedimentos, e de até $800 \mathrm{mg} \mathrm{kg}^{-1} \mathrm{em}$ solos, registrados por Matschullat (2000) no Quadrilátero Ferrífero, em Minas Gerais.

No solo, a sorção de oxiânions como o arsenato ocorre em óxidos e hidróxidos de $\mathrm{Fe}, \mathrm{Al}$ e $\mathrm{Mn}$ e argilossilicatos (McBride, 1989). A adsorção, a dessorção e a concentração do As na solução do solo são controladas pelos seus atributos físicos, químicos e biológicos. Nos atributos químicos, os fatores que influenciam as características de sorção são o pH da solução, a composição iônica e a força iônica. Na produção agrícola, esses parâmetros 
variam no tempo, em resposta às condições de manejo, climáticas, aplicações de fertilizantes, adubos orgânicos, calcário e gesso, e variam também no espaço, por causa da infiltração da água com menor força iônica que a solução do solo pelos macroporos (Jonge \& Jonge, 1999).

A avaliação do efeito da mudança na força iônica no comportamento da adsorção, fornece subsídios para inferir sobre o mecanismo de adsorção. Íons que formam complexos do tipo esfera externa mostram diminuição na adsorção com o aumento da força iônica, enquanto que íons que formam complexo de esfera interna apresentam aumento ou ausência de efeito na adsorção com o aumento da força iônica (McBride, 1997, citado por Goldberg \& Johnston, 2001). O aumento da força iônica resulta em aumento da adsorção do fosfato e do arsenato, quando o $\mathrm{pH}$ se encontra acima do ponto isoelétrico. A adsorção aumenta em razão da menor interação repulsiva entre as cargas negativas da superfície do colóide e as cargas negativas do íon, pois os íons eletrolíticos formam um escudo sobre a superfície carregada com alta concentração salina (Hiemstra \& Van Riemsdijk, 1999).

As interações químicas do arsênio no ambiente, aliadas às diferenças entre as propriedades dos compostos de origem natural e antropogênica, tornam a química ambiental do arsênio complexa (Barra et al., 2000). Estudos para o entendimento das interações químicas do arsênio em solos tropicais podem auxiliar na avaliação do risco de entrada desse elemento na cadeia alimentar, e na tomada de decisão sobre quando a remediação de certas áreas contaminadas minimizaria os possíveis danos às comunidades circunvizinhas. Apesar da importância da avaliação do efeito da força iônica na adsorção de arsênio, em solos de cargas variáveis, poucos estudos foram realizados nesta área (Smith et al., 1998).

Este trabalho teve por objetivo avaliar o efeito da força iônica da solução de equilíbrio, sobre a adsorção de As em latossolos de diferentes regiões geográficas brasileiras.

\section{Material e Métodos}

Foram utilizadas amostras da camada superficial (0-20 cm) de 17 latossolos, localizados em áreas experimentais de diversas regiões geográficas brasileiras. A localização e a classificação dos solos estudados, bem como a metodologia e os resultados da caracterização química, física e mineralógica podem ser encontrados em Pierangeli et al. (2001) e Silva et al. (1996).

Para a realização do experimento de adsorção, foram preparadas suspensões de terra fina seca ao ar (TFSA) dos 17 latossolos, em solução salina de $\mathrm{NaCl}$, em duas concentrações: $15 \mathrm{mmol} \mathrm{L}^{-1}$ e $150 \mathrm{mmol} \mathrm{L}^{-1}$. A força iônica de $15 \mathrm{mmol} \mathrm{L}^{-1}$ foi usada por ser semelhante à observada na solução de solos intemperizados. Todas as suspensões tiveram o $\mathrm{pH}$ ajustado a $5,5 \pm 0,2$, esse ajuste foi realizado utilizando-se $\mathrm{NaOH}$ e $\mathrm{HCl}$ a $10 \mathrm{mmol} \mathrm{L}^{-1}$. A relação solo:solução final foi de 1:100 e todas as amostras foram avaliadas em triplicata.

Depois da estabilização do $\mathrm{pH}$, foram adicionadas soluções de $\mathrm{Na}_{2} \mathrm{HAsO}_{4} .7 \mathrm{H}_{2} \mathrm{O}$ em $\mathrm{NaCl}$, para que fossem atingidas as concentrações iniciais de As de 15 e $150 \mu \mathrm{mol} \mathrm{L}^{-1}$ (1,1 e $11 \mathrm{mg} \mathrm{kg}^{-1}$, respectivamente). O tempo de contato foi de 72 horas, divididas em ciclos de 12 horas de agitação e 12 horas em repouso. Depois das 72 horas de reação, a suspensão de cada solo foi centrifugada a $8.000 \mathrm{rpm}$ por 20 minutos, e o sobrenadante foi retirado para leitura.

A dessorção foi conduzida em solução de fundo $(\mathrm{NaCl}) \mathrm{com}$ a mesma força iônica da adsorção (15 e $150 \mathrm{mmol} \mathrm{L}^{-1}$ ). O tempo de contato e coleta das alíquotas, para a determinação da quantidade de As dessorvida, seguiu a mesma metodologia da adsorção.

A quantidade adsorvida foi calculada, aplicando-se as seguintes equações:

$\mathrm{As}_{\mathrm{ads}}=\left\{(\mathrm{Ci}-\mathrm{Ce})\left[\left(\mathrm{V}_{1}+\mathrm{V}_{2}\right) / \mathrm{M}_{\mathrm{s}}\right]\right\}$

$\mathrm{As}_{\mathrm{adc}}=\left\{\left(\mathrm{Ci}\left[\left(\mathrm{V}_{1}+\mathrm{V}_{2}\right) / \mathrm{M}_{\mathrm{s}}\right]\right\} \mathrm{e}\right.$

$\% A s=\left(\mathrm{As}_{\text {ads }} / \mathrm{As}_{\mathrm{adc}}\right) 100$

em que $\mathrm{As}_{\text {ads }}$ é o arsênio adsorvido, em $\mathrm{mg} \mathrm{kg}^{-1}$ de solo; Ce é a concentração de equilíbrio depois de 72 horas, em mg L ${ }^{-1} ; M_{s}$ é a massa do solo, em g; As adc é o arsênio adicionado, em $\mathrm{mg} \mathrm{L}^{-1}$ de solo; Ci é a concentração inicial de As, em mg kg-1 ${ }^{-1} V_{1}$ é o volume, em mL, da solução padrão de arsênio para se atingir as concentrações de 15 e $150 \mu \mathrm{mol} \mathrm{L}^{-1}$; $\mathrm{V}_{2}$ é o volume, em mL, de solução de fundo ( $\mathrm{NaCl} 15$ ou $150 \mathrm{mmol} \mathrm{L}^{-1}$ ); e \%As é a porcentagem de arsênio adsorvido pelo solo.

Além do cálculo das quantidades de As adsorvidas e dessorvidas por cada solo, foi determinado também o coeficiente de distribuição de As (Kd, em L kg-1), nas diferentes amostras pela equação $\mathrm{Kd}=\mathrm{As}_{\mathrm{ads}} / \mathrm{Ce}$, porém, para melhor visualização dos valores de Kd, esses serão apresentados em base logarítmica.

A quantificação do teor de As, em todas as análises, foi realizada por espectrofotometria de absorção atômica, usando-se equipamento Perkin Elmer AAnalyst 800, com atomização eletrotérmica em forno de grafite. As condições de leitura utilizadas foram temperatura de pirólise de $500^{\circ} \mathrm{C}$ (recomendação do fabricante) e $0,003 \mathrm{mg}$ de $\mathrm{Mg}$ na forma de $\mathrm{Mg}\left(\mathrm{NO}_{3}\right)_{2}$, como modificador químico (Niedzielski et al., 2002). A análise de variância foi feita, utilizando-se o software 
SISVAR 4.3 (Ferreira, 2000). Para comparação entre médias utilizou-se o teste de Scott-Knott a 5\%.

\section{Resultados e Discussão}

A porcentagem média de adsorção, nos valores de força iônica (FI) 15 e $150 \mathrm{mmol} \mathrm{L}^{-1}$, foi de 63,51 e $64,46 \%$, respectivamente. Além da porcentagem média não ter sido diferente $(\mathrm{p}<0,05)$, observou-se também semelhanças entre amplitude total (88,02 e 88,65\%), máximos (99,59 e 97,83\%) e mínimos (11,57 e 9,18\%), o que evidencia que o aumento da força iônica não proporcionou alteração significativa na porcentagem de adsorção. Esses resultados concordam com o observado em estudo de adsorção de arsenato em óxidos de Fe amorfos, por Gao \& Mucci (2001) e Goldberg \& Johnston (2001).

Ao se considerar o efeito do aumento da FI, em cada solo, observou-se que apenas um solo (LVd4), na concentração $15 \mu \mathrm{mol} \mathrm{L}^{-1}$ de As e três solos (LVd4, LVd6 e LVef1), na concentração $150 \mu \mathrm{mol} \mathrm{L}^{-1}$ de As apresentaram diferença na porcentagem de adsorção entre valores de força iônica (Tabela 1). O aumento da força iônica proporcionou, nesses solos, elevação na porcentagem adsorvida, com exceção do solo LVd4 que apresentou elevação na adsorção com o aumento da FI, na dose $15 \mu \mathrm{mol} \mathrm{L}{ }^{-1}$ de As e diminuição na dose $150 \mu \mathrm{mol} \mathrm{L}^{-1} \mathrm{de}$ As. Ressalta-se que todos os solos sofreram ajuste do valor de $\mathrm{pH}$ para 5,5 $\pm 0,2$, e que esse valor encontra-se acima do ponto de efeito salino nulo (PESN) dos solos estudados (Silva et al., 1996). O comportamento de LVd4, na maior dose de arsênio, só seria justificável em valores de pH abaixo do PESN. O aumento da força iônica resulta em aumento da adsorção do fosfato e do arsenato, quando o pH encontra-se acima do ponto isoelétrico (Hiemstra \& Van Riemsdijk, 1999). A adsorção aumenta em razão da menor interação repulsiva entre as cargas negativas da superfície do colóide e as cargas negativas do íon, pois os íons eletrolíticos formam um escudo sobre a superfície carregada, em alta concentração salina. Esse efeito da força iônica sobre a adsorção, apresentado por Hiemstra \& Van Riemsdijk (1999), pode explicar o aumento na adsorção nos solos LVd4 (concentração de $15 \mu \mathrm{mol} \mathrm{L}{ }^{-1}$ de As), LVd6 e LVef1.

$\mathrm{O}$ coeficiente de distribuição $(\mathrm{Kd})$ de As, nos 17 latossolos estudados, não sofreu influência do aumento da força iônica (Tabela 1), exceto nos solos LVd2, LVd4, LVd6 e LVdf1 (concentração de $15 \mu \mathrm{mol} \mathrm{L}{ }^{-1}$ de As) e
LVd6 (concentração de $150 \mu \mathrm{mol} \mathrm{L}^{-1}$ de As). Nesses solos, o aumento da força iônica resultou em aumento do Kd, o que significa que o aumento da força iônica resultou em diminuição do arsênio em solução. Como o Kd é resultado da relação do As adsorvido pela concentração na solução no equilíbrio, valor de Kd alto significa que o adsorvato posssui grande afinidade com o adsorvente.

Os latossolos estudados apresentaram, nos dois valores de força iônica, porcentagem de dessorção média menor que $15 \%$, na menor dose de arsênio adicionada (Tabela 1), com exceção dos solos LVd4 e LAd1, que revelaram porcentagem de dessorção superior a $20 \%$, nos dois valores de força iônica. A porcentagem de

Tabela 1. Porcentagem adsorvida e dessorvida de arsênio e valor Log Kd, em amostras do horizonte A de latossolos brasileiros, em dois valores de força iônica (FI) da solução de equilíbrio (FI1, 15 mmol L-1; FI2, $150 \mathrm{mmol} \mathrm{L}^{-1}$ ) e duas concentrações de arsênio $\left(15 \text { e } 150 \mu \mathrm{mol} \mathrm{L}^{-1}\right)^{(1)}$.

\begin{tabular}{|c|c|c|c|c|c|c|}
\hline \multirow[t]{2}{*}{ Solo } & \multicolumn{2}{|c|}{ Adsorção (\%) } & \multicolumn{2}{|c|}{ Dessorção (\%) } & \multicolumn{2}{|c|}{$\log \mathrm{Kd}$} \\
\hline & FI1 & FI2 & FI1 & FI2 & FI1 & FI2 \\
\hline & \multicolumn{6}{|c|}{$15 \mu \mathrm{m} \mathrm{L}^{-1} \mathrm{de} A s$} \\
\hline LVd1 & $98,2 \mathrm{a}$ & $97,4 a$ & $0,8 b$ & $11,6 \mathrm{a}$ & $3,7 \mathrm{a}$ & $3,6 \mathrm{a}$ \\
\hline LVd2 & $93,4 a$ & $97,8 \mathrm{a}$ & $0,6 b$ & $6,6 \mathrm{a}$ & $3,1 b$ & $3,7 \mathrm{a}$ \\
\hline LVd3 & $84,2 \mathrm{a}$ & $85,9 \mathrm{a}$ & $0,9 b$ & $9,0 \mathrm{a}$ & $2,7 \mathrm{a}$ & $2,8 \mathrm{a}$ \\
\hline LVd4 & $31,1 b$ & $43,3 a$ & $33,7 \mathrm{a}$ & $36,0 \mathrm{a}$ & $1,6 \mathrm{~b}$ & $1,9 \mathrm{a}$ \\
\hline LVd5 & $63,3 \mathrm{a}$ & $67,4 a$ & $9,6 \mathrm{a}$ & $12,7 \mathrm{a}$ & $2,2 \mathrm{a}$ & $2,3 \mathrm{a}$ \\
\hline LVd6 & $93,2 \mathrm{a}$ & $96,6 a$ & $1,1 \mathrm{a}$ & $3,5 \mathrm{a}$ & $3,1 b$ & $3,5 \mathrm{a}$ \\
\hline LVd7 & $91,2 \mathrm{a}$ & $92,2 \mathrm{a}$ & $0,6 \mathrm{a}$ & $3,1 \mathrm{a}$ & $3,0 \mathrm{a}$ & $3,1 \mathrm{a}$ \\
\hline LVd8 & $91,2 \mathrm{a}$ & $93,1 \mathrm{a}$ & $2,8 b$ & $14,8 \mathrm{a}$ & $3,0 \mathrm{a}$ & $3,1 \mathrm{a}$ \\
\hline LVd9 & $91,1 \mathrm{a}$ & $91,4 a$ & $1,1 \mathrm{a}$ & $3,4 \mathrm{a}$ & $2,9 \mathrm{a}$ & $3,0 \mathrm{a}$ \\
\hline LVdf1 & $99,6 \mathrm{a}$ & $81,6 a$ & $0,1 \mathrm{~b}$ & $6,4 \mathrm{a}$ & $4,4 b$ & $2,6 a$ \\
\hline LVdf2 & $93,4 a$ & $93,3 a$ & $2,5 a$ & $6,0 \mathrm{a}$ & $3,1 \mathrm{a}$ & $3,1 \mathrm{a}$ \\
\hline LVdf3 & $97,8 \mathrm{a}$ & $93,9 a$ & $0,8 b$ & $6,6 \mathrm{a}$ & $3,6 \mathrm{a}$ & $3,2 \mathrm{a}$ \\
\hline LVef1 & $96,2 \mathrm{a}$ & $96,0 \mathrm{a}$ & $1,4 \mathrm{a}$ & $6,9 a$ & $3,4 \mathrm{a}$ & $3,5 \mathrm{a}$ \\
\hline LAd1 & $46,9 a$ & $49,2 \mathrm{a}$ & $17,8 \mathrm{a}$ & $25,0 \mathrm{a}$ & $1,9 \mathrm{a}$ & $1,9 \mathrm{a}$ \\
\hline LAd2 & $95,3 \mathrm{a}$ & $96,7 \mathrm{a}$ & $1,1 \mathrm{a}$ & $2,3 a$ & $3,3 \mathrm{a}$ & $3,5 \mathrm{a}$ \\
\hline LAd3 & $60,1 \mathrm{a}$ & $63,5 \mathrm{a}$ & $12,4 \mathrm{a}$ & $14,0 \mathrm{a}$ & $2,2 \mathrm{a}$ & $2,2 \mathrm{a}$ \\
\hline \multirow[t]{2}{*}{ LAx1 } & $60,9 a$ & $64,0 \mathrm{a}$ & $9,6 \mathrm{a}$ & $16,9 \mathrm{a}$ & $2,2 \mathrm{a}$ & $2,2 \mathrm{a}$ \\
\hline & \multicolumn{6}{|c|}{$150 \mu \mathrm{m} \mathrm{L}^{-1}$ de As } \\
\hline LVd1 & $65,5 \mathrm{a}$ & $65,7 \mathrm{a}$ & $24,0 \mathrm{a}$ & $13,4 a$ & $2,3 \mathrm{a}$ & $2,3 \mathrm{a}$ \\
\hline $\mathrm{LVd} 2$ & $60,4 a$ & $52,9 \mathrm{a}$ & $29,3 \mathrm{a}$ & $17,5 \mathrm{a}$ & $2,2 \mathrm{a}$ & $2,0 \mathrm{a}$ \\
\hline LVd3 & $58,1 \mathrm{a}$ & $52,8 \mathrm{a}$ & $31,3 \mathrm{a}$ & $17,6 \mathrm{a}$ & $2,1 \mathrm{a}$ & $2,0 \mathrm{a}$ \\
\hline LVd4 & $24,7 \mathrm{a}$ & $9,2 \mathrm{~b}$ & $79,8 \mathrm{a}$ & $86,2 \mathrm{a}$ & $1,5 \mathrm{a}$ & $0,9 b$ \\
\hline LVd5 & $23,4 \mathrm{a}$ & $15,3 \mathrm{a}$ & $83,7 \mathrm{a}$ & $59,6 \mathrm{a}$ & $0,8 \mathrm{a}$ & $1,2 \mathrm{a}$ \\
\hline LVd6 & $35,4 \mathrm{~b}$ & $55,4 \mathrm{a}$ & $60,1 \mathrm{a}$ & $12,7 \mathrm{~b}$ & $1,7 \mathrm{~b}$ & $2,1 \mathrm{a}$ \\
\hline LVd7 & $58,0 \mathrm{a}$ & $59,8 \mathrm{a}$ & $16,4 \mathrm{~b}$ & $25,8 \mathrm{a}$ & $2,1 \mathrm{a}$ & $2,2 \mathrm{a}$ \\
\hline LVd8 & $47,3 \mathrm{a}$ & $39,8 \mathrm{a}$ & $33,1 \mathrm{a}$ & $30,5 \mathrm{a}$ & $1,9 \mathrm{a}$ & $1,8 \mathrm{a}$ \\
\hline LVd9 & $47,6 \mathrm{a}$ & $49,9 \mathrm{a}$ & $22,8 \mathrm{a}$ & $32,8 \mathrm{a}$ & $1,9 \mathrm{a}$ & $1,9 \mathrm{a}$ \\
\hline LVdf1 & $83,0 \mathrm{a}$ & $76,9 a$ & $9,7 \mathrm{a}$ & $6,1 \mathrm{a}$ & $2,7 \mathrm{a}$ & $2,5 \mathrm{a}$ \\
\hline LVdf2 & $49,4 \mathrm{a}$ & $51,8 \mathrm{a}$ & $32,2 \mathrm{a}$ & $19,3 \mathrm{a}$ & $1,9 \mathrm{a}$ & $2,0 \mathrm{a}$ \\
\hline LVdf3 & $70,3 \mathrm{a}$ & $74,5 \mathrm{a}$ & $10,9 \mathrm{a}$ & $9,6 \mathrm{a}$ & $2,4 \mathrm{a}$ & $2,5 \mathrm{a}$ \\
\hline LVef1 & $37,4 \mathrm{~b}$ & $50,4 \mathrm{a}$ & $29,2 \mathrm{a}$ & $31,6 \mathrm{a}$ & $1,8 \mathrm{a}$ & $2,0 \mathrm{a}$ \\
\hline LAd1 & $15,5 \mathrm{a}$ & $15,6 \mathrm{a}$ & $77,2 \mathrm{a}$ & $70,0 \mathrm{a}$ & $1,3 \mathrm{a}$ & $1,2 \mathrm{a}$ \\
\hline LAd2 & $70,3 \mathrm{a}$ & $77,5 \mathrm{a}$ & $8,2 \mathrm{a}$ & $6,0 \mathrm{a}$ & $2,7 \mathrm{a}$ & $2,5 \mathrm{a}$ \\
\hline LAd3 & $11,6 \mathrm{a}$ & $23,6 \mathrm{a}$ & $73,4 a$ & $48,4 \mathrm{a}$ & $1,1 \mathrm{a}$ & $1,4 \mathrm{a}$ \\
\hline LAx1 & $14,3 \mathrm{a}$ & $17,1 \mathrm{a}$ & $56,7 \mathrm{a}$ & $56,8 \mathrm{a}$ & $1,0 \mathrm{a}$ & $1,3 \mathrm{a}$ \\
\hline
\end{tabular}

${ }^{(1)}$ Médias seguidas de mesma letra, na coluna, não diferem entre si pelo teste de Scott-Knott a 5\% de probabilidade. 
dessorção dos solos LVd4 e LAd1, aliada a seus menores teores de argila $\left(<350 \mathrm{~g} \mathrm{~kg}^{-1}\right)$ e de $\mathrm{Fe}_{2} \mathrm{O}_{3}\left(<80 \mathrm{~g} \mathrm{~kg}^{-1}\right)$, podem indicar predomínio de adsorção não específica de As.

Quando a dessorção foi avaliada na maior dose de arsênio adicionada, observou-se que os valores de porcentagem dessorvida foram, em média, superiores ao da menor dose de As ( $\left.15 \mathrm{mmol} \mathrm{L}^{-1}\right)$ e que o solo LVd6 apresentou diferença significativa na porcentagem dessorvida entre forças iônicas (Tabela 1).

A adsorção de As tem sido avaliada em sistemas puros e complexos, em vários valores de $\mathrm{pH}$ e força iônica, e na presença de íons competidores e, ao contrário da adsorção, quase não existem informações sobre o fenômeno de dessorção de As ou de outros oxiânions (Smith et al., 1998). Essa falta de estudos sobre dessorção de As dificulta a comparação entre os dados encontrados neste trabalho e os da literatura.

\section{Conclusão}

1. O aumento da força iônica não influencia a porcentagem de adsorção de As.

2. O aumento da força iônica, de 15 para $150 \mathrm{mmol} \mathrm{L}^{-1}$, não influencia a dessorção de arsênio, na maioria dos latossolos estudados.

\section{Agradecimentos}

A A.O.A. Camargo e F. Lombardi Neto (IAC), R.A. Dhein (Cotrijui), J.E. Denardin (Embrapa Trigo), M. Veiga (Epagri), C. Castro Filho (Iapar), L.C. Hernani e C.H. Kurihara (Embrapa Agropecuária Oeste), M. Martins Filho e V.P. Pereira (Unesp), D.P. Santana (Embrapa Milho e Sorgo), D.V.S. Resck (Embrapa Cerrados), I.F. Silva (UFPB), J.R.C. Silva (UFCE) e T.E. Rodrigues e L.D. Costa (Embrapa Solos), pelo envio das amostras de solos.

\section{Referências}

BAIRD, C. Química ambiental. 2.ed. Porto Alegre: Bookman, 2002. 621p.

BARRA, C.M.; SANTELLI, R.E.; ABRÃO, J.J.; GUARDIA, M. de la. Especiação de arsênio: uma revisão. Química Nova, v.23, p.58-70, 2000.

BASU, A.; MAHATA, J.; GUPTA, S.; GIRI, A.K. Genetic toxicology of a paradoxical human carcinogen, arsenic: a review. Mutation Research, v.488, p.171-194, 2001.
FERREIRA, D.F. Manual do sistema Sisvar para análises estatísticas. Lavras: Ufla, 2000. 63p.

GAO, Y.; MUCCI, A. Acid base reactions, phosphate and arsenate complexation, and their competitive adsorption at the surface of goethite in $0,7 \mathrm{M} \mathrm{NaCl}$ solution. Geochimica et Cosmochimica Acta, v.65, p.2361-2378, 2001.

GOLDBERG, S.; JOHNSTON, C.T. Mechanisms of arsenic adsorption on amorphous oxides evaluated using macroscopic measurements, vibrational spectroscopy, and surface complexation modeling. Journal of Colloid and Interface Science, v.234, p.204-216, 2001.

HIEMSTRA, T.; VAN RIEMSDIJK, W.H. Surface structural ion adsorption modeling of competitive binding of oxyanions by metal (hidr)oxides. Journal of Colloid and Interface Science, v.210, p.182-193, 1999.

JONGE, H. de; JONGE, L.W. de. Influence of $\mathrm{pH}$ and solution composition on the sorption of glyphosate and prochloraz to a sandy loam soil. Chemosphere, v.39, p.753-763, 1999.

McBRIDE, M.B. Reactions controlling heavy metal solubility in soils. Advances in Soil Sciences, v.10, p.1-56, 1989.

MATSCHULLAT, J. Arsenic in the geosphere: a review. Science of the Total Environmental, v.249, p.297-312, 2000.

NIEDZIELSKI, P.; SIEPAK, M.; SIEPAK, J. Comparison of modifiers for determination of arsenic, antimony and selenium by atomic absorption spectrometry with atomization in graphite tube or hydride generation and in-situ preconcentration in graphite tube. Microchemical Journal, v.72, p.137-145, 2002.

PIERANGELI, M.A.P.; GUILHERME, L.R.G.; CURI, N.; SILVA, M.L.N.; OLIVEIRA, L.R.; LIMA, J.M. de. Efeito do pH na adsorção-dessorção de chumbo em latossolos brasileiros. Revista Brasileira de Ciência do Solo, v.25, p.269-277, 2001.

ROYCHOWDHURY, T.; UCHINO, T.; TOKUNAGA, H.; ANDO, $M$. Arsenic and other heavy metals in soils from an arsenic-affected area of West Bengal, India. Chemosphere, v.49, p.605-618, 2002.

SILVA, M.L.N.; CURI, N.; MARQUES, J.J.G. de S. e M.; GUILHERME, L.R.G.; LIMA, J.M. de. Ponto de efeito salino nulo e suas relações com propriedades mineralógicas e químicas de latossolos brasileiros. Pesquisa Agropecuária Brasileira, v.31, p.663-671, 1996.

SMITH, E.; NAIDU, R.; ALSTON, A.M. Arsenic in the soil environment: a review. Advances in Agronomy, v.64, p.149-195, 1998.

UNITED STATES. Agency for Toxic Substances and Disease Registry. CERCLA priority list of hazardous substances. Atlanta, GA: U. S. Dept. of Health and Human Services, Public Health Service, 2003. Disponível em: <http://www.atsdr.cdc.gov/clist.html>. Acesso em: jun. 2004.

UNITED STATES. Agency for Toxic Substances and Disease Registry. Toxicological profile for arsenic. Atlanta, GA: U. S. Dept. of Health and Human Services, Public Health Service, 2000. 468p. 